\title{
Pharmacogenetic analysis of cinacalcet response in secondary hyperparathyroidism patients
}

This article was published in the following Dove Press journal:

Drug Design, Development and Therapy

8 July 2016

Number of times this article has been viewed

\author{
Sohyun Jeong' \\ In-Wha Kim' \\ Kook-Hwan $\mathrm{Oh}^{2}$ \\ Nayoung Han' \\ Kwon Wook Joo 2 \\ Hyo Jin Kim² \\ Jung $\mathrm{MiOh}{ }^{1}$
}

'College of Pharmacy, Research Institute of Pharmaceutical Sciences, Seoul National University,

${ }^{2}$ Department of Internal Medicine,

Division of Nephrology, Seoul

National University Hospital,

Seoul, Korea
Correspondence: Jung $\mathrm{Mi}$ Oh

College of Pharmacy, Research Institute of Pharmaceutical Sciences, Seoul National University, I Gwanakro, Gwanak-gu, Seoul 08826, Korea

$\mathrm{Tel}+8228807736$

Fax +82 28829560

Email jmoh@snu.ac.kr
Background: Secondary hyperparathyroidism (SHPT) is one of the major risk factors of morbidity and mortality in end-stage renal disease. Cinacalcet effectively controls SHPT without causing hypercalcemia and hyperphosphatemia. However, there is significant interindividual response variance to cinacalcet treatment. Therefore, we aimed to evaluate the genetic effects related with parathyroid hormone regulation as factors for cinacalcet response variance.

Methods: Patients with a diagnosis of SHPT based on intact parathyroid hormone (iPTH) $>300 \mathrm{pg} / \mathrm{mL}$ on dialysis were included in this study. They were over 18 years and have been treated by cinacalcet for more than 3 months. Responders and nonresponders were grouped by the serum iPTH changes. Twenty-four single nucleotide polymorphisms of CASR, $V D R, F G F R 1, K L, A L P L, R G S 14, N R 4 A 2$, and PTHLH genes were selected for the pharmacogenetic analysis.

Results: After adjusting for age, sex, and calcium level, CASR rs1042636 (odds ratio [OR]: $0.066, P=0.027)$ and $\mathrm{rs} 1802757$ (OR: $10.532, P=0.042$ ) were associated with cinacalcet response. The association of haplotypes of CASR rs1042636, rs10190, and rs1802757; GCC (OR: 0.355, $P=0.015$ ); and ATT (OR: 2.769, $P=0.014$ ) with cinacalcet response was also significant.

Conclusion: We obtained supporting information of the associations between cinacalcet response and CASR polymorphisms. CASR single nucleotide polymorphisms (SNPs) rs1802757, rs1042636, and haplotypes of rs1042636, rs 10190, and rs1802757 were significantly associated with cinacalcet response variance.

Keywords: $C A S R$, calcium sensing receptor, SHPT, genetic polymorphisms, haplotype, single nucleotide polymorphisms

\section{Introduction}

Dysregulation of mineral homeostasis due to failing kidney function leads to decreased renal phosphate excretion, elevated fibroblast growth factor 23 level, and reduced synthesis of calcitriol. These changes lead to consistent secretion of parathyroid hormone (PTH) contributing to the development of parathyroid hyperplasia and secondary hyperparathyroidism (SHPT) in end-stage renal disease. SHPT with elevated calcium and phosphate levels contributes to the development of renal osteodystrophy, erythropoietin resistance, vascular calcification, and left ventricular hypertrophy. These clinical features are strongly associated with increased morbidity and mortality in dialytic end-stage renal disease population. ${ }^{1-4}$ It is therefore critical to maintain optimal level of PTH in these patients. However, because of the complicated problems of lowering PTH with simultaneously controlling imbalanced serum levels of calcium and phosphate, traditional therapies of phosphate binders and vitamin D analogues for managing SHPT have all too often led to clinical problems, including hypercalcemia, 
hyperphosphatemia, and refractory hyperparathyroidism or over-suppression of PTH. ${ }^{5}$

One of the newer treatment options for SHPT is cinacalcet which increases the sensitivity of the calcium-sensing receptor (CaSR) on the parathyroid gland cells to extracellular calcium, ${ }^{6}$ resulting in a decreased calcium set point and circulating PTH levels. ${ }^{6-8}$ The cinacalcet treatment resulted in $41 \%$ of SHPT patients achieving PTH and $\mathrm{Ca} \times \mathrm{P}$ levels recommended by the Kidney Disease Outcomes Quality Initiative (K/DOQI) guidelines ${ }^{9}$ whereas fewer than $10 \%$ of the patients treated with phosphate binders and vitamin $\mathrm{D}$ analogs reached optimal control. ${ }^{10}$ Moreover, cinacalcet reduced the risk of parathyroidectomy by $91 \% .{ }^{11}$ Cinacalcet treatment is effective to the extent that it can be utilized as an alternative to surgical intervention in some patients. ${ }^{12,13}$ Despite the overall response to cinacalcet being promising in a number of clinical trials, ${ }^{14-16}$ the therapeutic efficacy has a significant inter-individual variability of $12.1 \%-46 \%$ PTH reduction, representing drug resistance. ${ }^{17-21}$ The factors associated with the drug resistance include delayed therapy, persistent hyperphosphatemia, nodular hyperplasia, ${ }^{22}$ and reduced expression of CaSR and vitamin D receptor (VDR). ${ }^{23-25}$ In particular, genetic polymorphisms affecting CaSR and VDR expression have been considered as factors for drug resistance. The genetic polymorphism of CASR Arg990Gly (c.2968A $>$ G, rs1042636) was associated with different cinacalcet response. ${ }^{26}$ Several other studies ${ }^{27-29}$ on $C A S R$ presented the possible association of CASR rs1042636 and rs2221266 polymorphisms with PTH level difference. VDR gene polymorphisms of rs7975232 (Apa 1), rs731236 (Taq 1), and rs1544410 (Bsm 1) were also associated with PTH regulation. ${ }^{30-32}$ These studies presented the clinical importance of genetic polymorphisms in PTH regulation. However, the results available so far show limited evidence for genetic polymorphisms associated with cinacalcet response involving a small number of patients and not including phosphate and bone regulation-related genes in PTH homeostasis. Thus, we aimed to evaluate the frequency of variants of study genes related to PTH regulation and the association between SNPs and haplotypes of genes and cinacalcet response in Korean SHPT patients on dialysis.

\section{Methods}

\section{Study subjects}

Study patients were selected from a tertiary university hospital in Seoul, Korea, between June 2011 and July 2014. Patients with a diagnosis of SHPT based on intact parathyroid hormone (iPTH) $>300 \mathrm{pg} / \mathrm{mL}$ were included in this study.
The patients were over 18 years and have been treated by cinacalcet for more than 3 months. All these patients were on either hemodialysis or peritoneal dialysis, receiving optimal medical therapy in the form of dietary restriction, phosphate binders, or vitamin D sterols. The main exclusion criteria were serious concomitant hepatic disease, active cancers, or taking strong CYP3A4 inhibitors, such as itraconazole, clarithromycin, diltiazem, and verapamil.

The study complies with the Helsinki Declaration and was approved by the Ethics Committee of the Seoul National University Hospital (IRB \#: H-1408-082-604). Written informed consents were obtained from all patients.

\section{Data collection}

Demographic information on the cause of chronic kidney disease (CKD), mode and duration of dialysis, concomitant drugs, and biochemical parameter levels of serum calcium, phosphorus, alkaline phosphatase, iPTH, creatinine, estimated glomerular filtration rate using Modification of Diet in Renal Disease equation, albumin, and hemoglobin at the start and during 3 months of cinacalcet treatment was obtained. Responders and nonresponders were grouped by their serum iPTH changes. Nonresponders were grouped as the patients whose iPTH levels increased even after 3 months of cinacalcet treatment. ${ }^{33,34}$ Otherwise, those patients whose iPTH values showed any reduction during the period of 3-month cinacalcet treatment were defined as responders.

Serum calcium and phosphate levels were measured by standard methods; iPTH was measured using a doubleantibody immunoradiometric assay for the quantitative determination of intact biological chain of 84 amino acids of PTH in human serum (ELSA PTH, Cisbio Bioassays, Codolet, France). Serum calcium was reported as albumin corrected values throughout this research.

\section{Genotype selection and analysis}

Twenty-five SNPs in nine genes which are previously known as related to calcium, phosphate, and bone metabolism were selected from the results of previous literatures and databases. Of these genes, $C A S R^{26-29,35-37}$ and $V D R^{30-32,38,39}$ involved in calcium regulation; FGFR $1,{ }^{40,41} \mathrm{KL},{ }^{42} R G S 14,{ }^{43,44}$ and $S L C 34 A 1^{45}$ related to phosphate serum concentration; and $A L P L,{ }^{46,47} N R 4 A 2,{ }^{48,49}$ and $P T H L H^{50-53}$ related to bone formation were evaluated. Peripheral blood samples were collected in ethylenediaminetetraacetic acid (EDTA) containing tubes and stored at $-80^{\circ} \mathrm{C}$ before DNA isolation. The SNaPshot assay was performed according to the manufacturer's instructions (ABI PRISM SNaPShot Multiplex kit, Thermo Fischer 
Scientific, Waltham, MA, USA). Table S1 shows the included SNPs and the primer sets and melting temperature used for the SNaPshot assay. Twenty-four studied SNPs of CASR, VDR, FGFR1, KL, ALPL, RGS14, NR4A2, and PTHLH were in Hardy-Weinberg Equilibrium, but SLC34A1 rs3812035 was not. Thus, association analysis of SLC $34 A 1$ was excluded.

\section{Statistical analysis}

The Hardy-Weinberg Equilibrium of each SNP was tested using the goodness-of-fit chi-square test to compare the expected frequencies of genotypes in controls; SNPs with $P>0.05$ were considered to be in Hardy-Weinberg Equilibrium. The independent-samples $t$-test and MannWhitney test were used to determine the differences between parametric and nonparametric characteristics of patients and biochemical parameter changes. The chi-square or Fisher's exact test was performed to assess the difference between categorical variables of the patient characteristics. Unconditional logistic regression analysis and chi-square or Fisher's exact test were used to evaluate the frequency analysis of polymorphisms of the genes and the relationships between different genotypes and cinacalcet response. Statistical analysis was carried out using IBM SPSS Statistics 21.0 for Windows (SPSS Inc., IBM Corporation, Armonk, NY, USA). Haplotypes and haplotype frequencies were calculated using Haploview software (version 4.2, Massachusetts Institute of Technology, Cambridge, MA, USA). The haplotype with $P<0.05$ was considered statistically significant.

\section{Results \\ Patient data}

A total of 70 dialysis patients with SHPT were included in the biochemical parameter analysis. Baseline characteristics were well balanced between the responders and nonresponders except for baseline calcium levels $(P=0.001)$. The baseline iPTH for 68 patients was $622(300-1,493) \mathrm{pg} / \mathrm{mL}$ versus $601(316-1,183) \mathrm{pg} / \mathrm{mL}(P=0.375)$ for responders versus nonresponders, respectively. The parathyroid gland hyperplasia or adenomatous state did not differ between two groups $(P=0.726)$. The amount of vitamin $\mathrm{D}$ (calcitriol or paricalcitol) taken by the patients $(P=0.116)$ and the number of patients on phosphate binders and vitamin $\mathrm{D}$ analogues were not different between the two groups $(P=0.116$ and $P=1.000$, respectively) (Table 1). The maintenance dose of cinacalcet ranged from 12.5 to $100 \mathrm{mg}$ per day, depending on patient's status. The dose was higher in nonresponders: responders $25(12.5-75) \mathrm{mg} /$ day versus nonresponders 50 $(25-100) \mathrm{mg} /$ day $(P<0.001)$.

\section{Biochemical parameter changes}

The biochemical parameter changes between baseline and 3-month treatment are summarized in Table 2. Cinacalcet significantly reduced serum iPTH levels in 53 responders. The percent iPTH changes between baseline and 3-month treatment were -42.29 (-97.41 to 9.23$) \%$ versus 6.16 (0.90 to 45.25$) \%$ in responders versus nonresponders, respectively $(P<0.001)$. At 3 -month treatment, $39.6 \%$ $(21 / 53)$ of responders had an iPTH level below $300 \mathrm{pg} / \mathrm{mL}$, the level recommended by the K/DOQI guidelines. ${ }^{9}$ The percent change of serum calcium concentration at 3-month treatment was $-5.75(-25.67$ to 15.81$) \%$ in responders compared to -7.13 ( -19.09 to 1.67$) \%$ in nonresponders $(P=0.182)$. The percent change of serum phosphorus reduction in responders was -9.84 (-69.23 to 69.43$) \%$ compared to -8.42 ( -33.33 to 72.43$) \%$ in nonresponders $(P=0.170)$.

\section{Frequency analysis of genetic polymorphisms}

The loci, allele, and minor allele frequency (MAF) results are summarized in Table 3. The observed MAFs in CASR rs 1042636 and rs 1802757 were $45.8 \%$ and $35.8 \%$, respectively. VDR rs7975232 (Apa l) and rs1544410 (bsm l) were $16.0 \%$ and $6.3 \%$, respectively, whereas rs 2228570 (Fok 1 ) was $38.2 \%$.

\section{Association with cinacalcet response Association of 24 SNPs with iPTH}

The association of 24 SNPs with cinacalcet response was evaluated (Table 4). The chi-square test showed that CASR rs 1042636 had a significant difference in genotype frequencies between responder and nonresponders $(P=0.036)$ but the MAF was not significant $(P=0.075)$. The chi-square test also proved that $C A S R$ rs 1042636 had a significant association with cinacalcet response (odds ratio [OR]: $0.267, P=0.035$ ) in the dominant genetic model (Table 5). Unconditional logistic regression analysis showed that $C A S R$ rs 1042636 (OR: 0.074, $P=0.025)$ (95\% confidence interval [CI]: 0.008-0.721) and rs 1802757 (OR: 8.625, $P=0.042)(95 \%$ CI: $1.077-69.075)$ had an association with the cinacalcet response in the genotype model, indicating that the A to G substitution of rs 1042636 decreased the risk of nonresponse by $93 \%$, whereas the $\mathrm{C}$ to T substitution of rs 1802757 increased the risk of nonresponse by approximately eight times. After adjusting for age, sex, and baseline calcium level, CASR rs1042636 (OR: 0.066, $P=0.027$ ) and rs 1802757 (OR: 10.532, $P=0.042$ ) were associated with cinacalcet response. 
Table I Demographic characteristics at baseline for included subjects $(n=70)$

\begin{tabular}{|c|c|c|c|c|}
\hline \multirow[t]{2}{*}{ Characteristics } & \multirow[t]{2}{*}{$\begin{array}{l}\text { Total } \\
(\mathbf{N}=70)^{*}(\%)\end{array}$} & \multicolumn{2}{|c|}{$\begin{array}{l}\text { Grouped by cinacalcet response } \\
(\mathrm{N}=68)^{* *}(\%)\end{array}$} & \multirow[t]{2}{*}{$P$-value } \\
\hline & & Responders $(n=53)$ & Nonresponders $(n=15)$ & \\
\hline Male sex, $\mathrm{n}(\%)$ & $32(45.7)$ & $27(50.9)$ & $4(26.7)$ & 0.096 \\
\hline Age, years, median (range) & $50(21-75)$ & $49(2 \mid-75)$ & $52(24-7 I)$ & 0.325 \\
\hline $\mathrm{BMI}, \mathrm{kg} / \mathrm{cm}^{2}$, median (range) & $22.37(16.01-31.45)$ & $22.08(16.01-31.00)$ & $23.29(17.76-27.38)$ & 0.206 \\
\hline \multicolumn{5}{|l|}{ Cause of CKD, n (\%) } \\
\hline DM & $12(17.2)$ & $7(13.2)$ & $4(26.7)$ & 0.680 \\
\hline HTN & $10(14.3)$ & $7(13.2)$ & $2(13.3)$ & 1.000 \\
\hline GN & $28(40.0)$ & $22(4 \mid .5)$ & $6(40.0)$ & 0.916 \\
\hline Others & $20(28.6)$ & $17(32.1)$ & $3(20.0)$ & 0.762 \\
\hline \multicolumn{5}{|l|}{ Mode of dialysis, $\mathrm{n}(\%)$} \\
\hline $\mathrm{HD}$ & $19(27.1)$ & II (20.8) & $7(46.7)$ & 0.093 \\
\hline PD & $40(57.1)$ & $33(62.3)$ & $6(40.0)$ & 0.124 \\
\hline Both & II (I5.7) & $9(17.0)$ & $2(13.3)$ & 1.000 \\
\hline Dialysis duration, median (range) & $97(38-195)$ & $77.5(15-300)$ & $97(38-195)$ & 0.363 \\
\hline $\begin{array}{l}\text { Use of phosphate binder/calcium } \\
\text { supplement, } n(\%)\end{array}$ & $62(88.6)$ & $48(90.6)$ & $14(93.3)$ & 1.000 \\
\hline \multicolumn{5}{|l|}{ Lab values, median (range) } \\
\hline iPTH $(\mathrm{pg} / \mathrm{mL})^{*}$ & $620(300-1,493)$ & $622(300-1,493)$ & $601(316-1,183)$ & 0.375 \\
\hline Albumin corrected calcium (mg/dL) & $9.84(7.4-13.52)$ & $9.72(7.40-13.52)$ & $10.50(9.18-11.88)$ & 0.001 \\
\hline Phosphorus (mg/dL) & $5.85(3.40-12.80)$ & $5.70(3.50-12.80)$ & $6.00(3.40-7.50)$ & 0.673 \\
\hline $\mathrm{Ca} \times \mathrm{P}\left(\mathrm{mg}^{2} / \mathrm{dL}^{2}\right)$ & $58.35(32.4 I-94.72)$ & $57.0 I(32.4 I-94.72)$ & $61.80(37.38-71.10)$ & 0.787 \\
\hline ALP (IU/L) & $106(4 I-393)$ & $100(4 \mid-38 I)$ & $124(6 \mid-762)$ & 0.252 \\
\hline $\mathrm{Hb}(\mathrm{g} / \mathrm{dL})$ & $10.50(7.00-14.60)$ & $10.70(7.00-14.00)$ & $10.10(7.70-14.60)$ & 0.107 \\
\hline Albumin (g/dL) & $3.80(2.40-11.50)$ & $3.80(2.40-5.10)$ & $3.90(3.10-4.40)$ & 0.953 \\
\hline Serum creatinine $(\mathrm{mg} / \mathrm{dL})$ & I I.69 (4.30-20.66) & $12.03(4.30-20.66)$ & $10.85(4.35-18.12)$ & 0.248 \\
\hline GFR $\left(\mathrm{mL} / \mathrm{min} / \mathrm{I} .73 \mathrm{~m}^{2}\right)$ & $3.80(2.10-15.7)$ & $3.80(2.50-13.30)$ & $3.80(2.10-15.70)$ & 0.842 \\
\hline \multicolumn{5}{|l|}{ Comorbidity related with CKD-MBD, n (\%) } \\
\hline Osteoporosis & $6(8.6)$ & $5(9.4)$ & I (6.7) & 1.000 \\
\hline Osteopenia & $22(3 \mid .4)$ & $18(34.0)$ & $3(20.0)$ & 0.361 \\
\hline Fracture & II (I5.7) & $7(13.2)$ & $4(26.7)$ & 0.243 \\
\hline PTH hyperplasia or adenoma & $15(21.4)$ & $12(22.6)$ & $3(20.0)$ & 0.726 \\
\hline HTN & 51 (72.9) & $39(73.6)$ & $10(66.7)$ & 0.745 \\
\hline IHD, HF, AF, LVH & $25(35.7)$ & $16(30.2)$ & $8(53.3)$ & 0.666 \\
\hline Comorbidity related with neoplasm & $13(18.6)$ & $7(13.2)$ & $5(33.3)$ & 0.442 \\
\hline
\end{tabular}

Notes: *A total of 70 patients were included in the final analysis. **A total of 68 patients who had available iPTH level were included in the cinacalcet response analysis. Significant results are marked in bold.

Abbreviations: AF, atrial fibrillation; ALP, alkaline phosphatase; BMI, body mass index; CKD, chronic kidney disease; CKD-MBD, chronic kidney disease-mineral bone disease; DM, diabetes mellitus; GFR, glomerular filtration rate; Hb, hemoglobin; HF, heart failure; HTN, hypertension; GN, glomerulonephritis; HD, hemodialysis; IHD, ischemic heart disease; iPTH, intact parathyroid hormone; LVH, left ventricular hypertrophy; PD, peritoneal dialysis; PTH, parathyroid hormone.

\section{Association of CASR haplotypes with iPTH}

The distribution of haplotypes was constructed for three genes and assessed for the association with the cinacalcet response. Linkage disequilibrium structures were designated by the $D^{\prime}$ value shown in Figure 1. Each of $C A S R, V D R$, and $A L P L$ genes included one haplotype block. The correlation coefficient $\left(r^{2}\right)$ between rs1042636 and rs1802757, rs1042636 and rs10190, and rs1802757 and rs10190 was

Table 2 Comparison of biochemical parameter percent changes between baseline and 3-month treatment with cinacalcet ( $\mathrm{n}=68$ )

\begin{tabular}{|c|c|c|c|}
\hline \multirow[t]{2}{*}{ Parameter } & \multirow{2}{*}{$\begin{array}{l}\text { Responders } \\
\text { Median (range) (\%) }\end{array}$} & \multirow{2}{*}{$\begin{array}{l}\text { Nonresponders } \\
\text { Median (range) (\%) }\end{array}$} & \multirow[t]{2}{*}{$P$-value* } \\
\hline & & & \\
\hline$\Delta \mathrm{PTH}(\mathrm{pg} / \mathrm{mL})$ & $-42.29(-97.4 I-[-9.23])$ & $6.16(0.90-45.25)$ & $<0.001$ \\
\hline$\Delta$ Calcium (mg/dL) & $-5.75(-25.67-15.81)$ & $-7.13(-19.09-1.67)$ & 0.182 \\
\hline$\Delta$ Phosphorus (mg/dL) & $-9.84(-69.23-69.43)$ & $-8.42(-33.33-72.43)$ & 0.170 \\
\hline$\Delta \mathrm{Ca} \times \mathrm{P}\left(\mathrm{mg}^{2} / \mathrm{dL}^{2}\right)$ & $-14.70(-71.13-68.70)$ & $-8.84(-36.99-43.70)$ & 0.296 \\
\hline$\Delta \mathrm{ALP}(\mathrm{IU} / \mathrm{L})$ & I.60 (-28.48-|46.58) & $|0.7|(-29.26-93.89)$ & 0.211 \\
\hline
\end{tabular}

Notes: The 3-month treatment value for biochemical parameters is defined as the mean value during baseline to 3 -month treatment. *Descriptive $P$-values were obtained from the independent-samples $t$-test or Mann-Whitney $U$-test. Significant results are marked in bold.

Abbreviations: ALP, alkaline phosphatase; PTH, parathyroid hormone. 
Table 3 The loci and minor allele frequency (MAF) information of the 24 SNPs in CASR, VDR, FGFRI, KL, RGSI4, SLC34AI, ALPL, NR4A2, and PTHLH genes

\begin{tabular}{|c|c|c|c|c|c|}
\hline Gene/SNP & $\begin{array}{l}\text { Chromosome } \\
\text { position }\end{array}$ & Alleles & $\begin{array}{l}\text { SNP } \\
\text { location }\end{array}$ & $\begin{array}{l}\text { Observed MAFI } \\
\text { expected MAF }\end{array}$ & $\begin{array}{l}\text { HWE test } \\
\text { (P-value) }\end{array}$ \\
\hline \multicolumn{6}{|l|}{ CASR } \\
\hline rsl042636 & 3:122284922 & $\mathrm{A} / \mathrm{G}$ & Exon & $0.458 / 0.2065$ & 0.59 \\
\hline rs2221266 & $3: 122277969$ & $\mathrm{C} / \mathrm{T}$ & Intron & $0.410 / 0.4619$ & 0.97 \\
\hline rs 10190 & $3: 122286389$ & $\mathrm{C} / \mathrm{T}$ & $3^{\prime} U T R$ & $0.387 / 0.4669$ & 0.81 \\
\hline rs|802757 & $3: 122286284$ & $\mathrm{C} / \mathrm{T}$ & $3^{\prime} U T R$ & $0.359 / 0.1983$ & 0.43 \\
\hline \multicolumn{6}{|l|}{ VDR } \\
\hline rs7975232 & I2:47845054 & $\mathrm{A} / \mathrm{C}$ & Intron & $0.160 / 0.4846$ & 0.87 \\
\hline rs2228570 & |2:47879| |2 & $\mathrm{C} / \mathrm{T}$ & Exon & $0.382 / 0.3285$ & 0.80 \\
\hline rsI5444I0 & $12: 47846052$ & $\mathrm{~A} / \mathrm{G}$ & Intron & $0.063 / 0.2959$ & 0.57 \\
\hline rsII574I 29 & $12: 47843520$ & $\mathrm{~T} / \mathrm{C}$ & $3^{\prime} U T R$ & $0.099 / 0.0385$ & 0.08 \\
\hline rs3847987 & $12: 47844285$ & $\mathrm{G} / \mathrm{T}$ & $3^{\prime}$ UTR & $0.106 / 0.1288$ & 0.13 \\
\hline rs739837 & $12: 47844438$ & C/A & $3^{\prime} U T R$ & $0.176 / 0.4942$ & 0.67 \\
\hline rs9729 & $12: 47842840$ & C/A & $3^{\prime} U T R$ & $0.169 / 0.4902$ & 0.71 \\
\hline \multicolumn{6}{|l|}{ FGFRI } \\
\hline rs13317 & $8: 38411996$ & $\mathrm{C} / \mathrm{T}$ & $3^{\prime}$ UTR & $0.345 / 0.2250$ & 0.06 \\
\hline \multicolumn{6}{|l|}{$K L$} \\
\hline rs|207568 & 13:330|6046 & $\mathrm{C} / \mathrm{T}$ & Exon & $0.160 / 0.1601$ & 0.11 \\
\hline \multicolumn{6}{|l|}{$R G S / 4$} \\
\hline rs4074995 & 5:177370342 & $\mathrm{A} / \mathrm{G}$ & Intron & $0.236 / 0.1895$ & 0.20 \\
\hline \multicolumn{6}{|l|}{ SLC34AI* } \\
\hline rs38I2035 & $5: 177390142$ & $\mathrm{~A} / \mathrm{C}$ & $3^{\prime}$ UTR & $0.255 / 0.387$ & 0.01 \\
\hline \multicolumn{6}{|l|}{$A L P L$} \\
\hline rsl69742I & I:21496799 & $\mathrm{A} / \mathrm{G}$ & Exon & $0.458 / 0.3776$ & 0.50 \\
\hline rs3200254 & $1: 21568242$ & $\mathrm{C} / \mathrm{T}$ & Exon & $0.424 / 0.2670$ & 0.66 \\
\hline rs2242420 & $1: 21578036$ & $\mathrm{C} / \mathrm{T}$ & $3^{\prime}$ UTR & $0.155 / 0.1172$ & 0.47 \\
\hline rs224242I & $|: 2157808|$ & $\mathrm{A} / \mathrm{G}$ & $3^{\prime}$ UTR & $0.345 / 0.2220$ & 0.46 \\
\hline rsl697405 & $1: 215777 \mid 3$ & $\mathrm{G} / \mathrm{A}$ & $3^{\prime}$ UTR & $0.268 / 0.3367$ & 0.43 \\
\hline rsl697406 & I:21577774 & $\mathrm{A} / \mathrm{G}$ & $3^{\prime}$ UTR & $0.148 / 0.1318$ & 0.49 \\
\hline \multicolumn{6}{|l|}{$N R 4 A 2$} \\
\hline$r s \mid 2803$ & $2: 156329935$ & $\mathrm{G} / \mathrm{A}$ & $3^{\prime}$ UTR & $0.2863 / 0.169$ & 0.45 \\
\hline \multicolumn{6}{|l|}{ PTHLH } \\
\hline rs2796 & $|2: 27963| 78$ & $\mathrm{~A} / \mathrm{G}$ & $3^{\prime}$ UTR & $0.127 / 0.0727$ & 0.76 \\
\hline rs 6253 & $12: 27963008$ & $\mathrm{~A} / \mathrm{G}$ & $3^{\prime}$ UTR & $0.394 / 0.3243$ & 0.09 \\
\hline rs 6245 & $|2: 27963| 24$ & $\mathrm{~A} / \mathrm{G}$ & $3^{\prime}$ UTR & $0.085 / 0.0629$ & 0.40 \\
\hline
\end{tabular}

Note: *Hardy-Weinberg Equilibrium (HWE) test was not satisfied and SLC34AI rs38I2035 excluded for our analysis. Significant results are marked in bold. Abbreviations: UTR, untranslated region; SNP, single nucleotide polymorphism; MAF, minor allele frequency.

$0.68,0.77$, and 0.87 , respectively, and showed high correlations. The CASR haplotypes of GCC (OR: 0.355, $P=0.015$ ) and ATT (OR: 2.769, $P=0.014$ ) were associated with cinacalcet response. With regard to the haplotypes of $V D R$ and $A L P L$, no significant association with cinacalcet response was found (Table 6).

\section{Association of CASR genotypes with biochemical parameters}

The associations between CASR genotypes and biochemical parameter changes were evaluated in 70 patients. The parameters showed that 3-month cinacalcet treatment lowered overall levels of serum calcium, $-6.64(-19.09$ to 15.81$)$ $\mathrm{mg} / \mathrm{dL}$, phosphate, -8.13 (-43.69 to 69.43$) \mathrm{mg} / \mathrm{dL}$, and $\mathrm{Ca} \times \mathrm{P},-12.22(-44.87$ to 68.70$) \mathrm{mg}^{2} / \mathrm{dL}^{2}$ but the level of alkaline phosphatase, 2.36 (-28.48 to 146.58$) \mathrm{mg} / \mathrm{dL}$ was increased. None of the genotypes of CASR was associated with the biochemical parameter changes (Table S2).

\section{Discussion}

In this study, the genotype frequencies of the SNPs related to PTH regulation and the association with cinacalcet response were evaluated. The observed MAFs in CASR rs 1042636 and rs 1802757 were double the reported frequency in HapMap $^{54}$ whereas VDR rs7975232 (Apa I) and rs1544410 (bsm I) showed a lower frequency than that of HapMap. The rs2228570 (Fok 1) showed a similar frequency. Furthermore, the novel findings of our study are that genetic polymorphisms of CASR rs 1802757 besides rs 1042636 and haplotypes of rs1042636, rs10190, and rs1802757 were significantly associated with cinacalcet response. The gain of function nonsynonymous SNP of rs1042636, which is 
Table 4 Comparisons of genotypes and allele frequencies between responders versus nonresponders group

\begin{tabular}{|c|c|c|c|c|c|c|}
\hline \multirow[t]{3}{*}{ Gene/SNP } & \multicolumn{3}{|c|}{ Genotype frequency (\%) } & \multicolumn{3}{|c|}{ Minor allele frequency (\%) } \\
\hline & \multirow{2}{*}{$\frac{\text { Responders }(n=53)}{\text { AA/AB/BB }}$} & \multirow{2}{*}{$\begin{array}{l}\text { Nonresponders }(n=15) \\
\text { AA/AB/BB }\end{array}$} & \multirow[t]{2}{*}{$P$-value } & \multirow{2}{*}{$\begin{array}{l}\text { Responders }(n=53) \\
B\end{array}$} & \multirow{2}{*}{$\begin{array}{l}\text { Nonresponders }(n=15) \\
\text { B }\end{array}$} & \multirow[t]{2}{*}{$P$-value } \\
\hline & & & & & & \\
\hline \multicolumn{7}{|l|}{ CASR } \\
\hline $\mathrm{rs} 1042636(\mathrm{~A} / \mathrm{G})$ & $|5| / 50.9 / 34.0$. & $40 / 53.3 / 6.7$ & 0.036 & 59.4 & 33.3 & 0.075 \\
\hline rs222I $266(\mathrm{C} / \mathrm{T})$ & $41.5 / 45.3 / 13.2$ & |3.3/53.3/33.3 & 0.086 & 35.8 & 60.0 & 0.111 \\
\hline 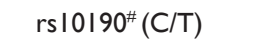 & $42.3 / 44.2 / 13.5$ & $15.4 / 6|.5 / 23|$. & 0.190 & 35.6 & 53.8 & 0.149 \\
\hline $\mathrm{rs} 1802757^{\#}(\mathrm{C} / \mathrm{T})$ & $44.2 / 48.1 / 7.7$ & $|5.4 / 6| .5 / 23 . \mid$ & 0.086 & 31.7 & 53.8 & 0.085 \\
\hline \multicolumn{7}{|l|}{ VDR } \\
\hline rs7975232 (G/T) & $66.0 / 28.3 / 3.8$ & $80 / 20 / 0$ & 0.336 & 17.9 & 10 & 0.292 \\
\hline rs2228570 (C/T) & $37.7 / 47.2 / 15.1$ & $33.3 / 60.0 / 6.7$ & 0.585 & 38.7 & 36.7 & 0.920 \\
\hline $\mathrm{rs} 1544410(\mathrm{~A} / \mathrm{G})$ & $84.9 / 15.1 / 0$ & $93.3 / 6.7 / 0$ & 0.672 & 7.5 & 3.3 & 1.000 \\
\hline rsII574|29\#(T/C) & $82.7 / 13.5 / 3.8$ & $84.6 / 15.4 / 0$ & 0.574 & 10.5 & 7.7 & 1.000 \\
\hline rs $3847987^{\#}(\mathrm{G} / \mathrm{T})$ & $78.8 / 15.4 / 3.8$ & $84.6 / 15.4 / 0$ & 0.706 & 11.5 & 7.7 & 0.672 \\
\hline rs739837" $(\mathrm{C} / \mathrm{A})$ & $61.5 / 32.7 / 3.8$ & $76.9 / 23.1 / 0$ & 0.762 & 20.2 & 11.5 & 1.000 \\
\hline rs9729" (C/A) & $63.5 / 30.8 / 3.8$ & $76.9 / 23.1 / 0$ & 0.769 & 19.2 & 11.5 & 1.000 \\
\hline \multicolumn{7}{|l|}{ FGFRI } \\
\hline rs13317 (G/A) & $47.2 / 39.6 / 13.2$ & $46.7 / 20.0 / 33.3$ & 0.313 & 33.0 & 43.3 & 0.865 \\
\hline \multicolumn{7}{|l|}{$K L$} \\
\hline rsI207568 (C/T) & $66.0 / 32.1 / 1.9$ & $66.7 / 33.3 / 0$ & 0.865 & 17.0 & 20.0 & 0.852 \\
\hline \multicolumn{7}{|l|}{ RGSI4 } \\
\hline rs4074995 (A/G) & $50.9 / 30.2 / 11.3$ (F:7.6) & $60.0 / 33.3 / 6.7$ & 0.828 & 26.4 & 23.3 & 1.000 \\
\hline \multicolumn{7}{|l|}{$A L P L$} \\
\hline rsl69742I (A/G) & $34.0 / 41.5 / 24.5$ & $6.7 / 46.7 / 26.7$ & 0.491 & 45.3 & 50.0 & 0.666 \\
\hline rs3200254 (C/T) & $30.2 / 45.3 / 24.5$ & $26.7 / 53.3 / 6.7(F: \mid 3.3)$ & 0.837 & 47.2 & 33.3 & 0.818 \\
\hline $\mathrm{rs} 2242420^{\#}(\mathrm{C} / \mathrm{T})$ & $65.4 / 32.7 / 1.9$ & $76.9 / 23.1 / 0$ & 0.880 & 18.3 & 11.5 & 1.000 \\
\hline $\mathrm{rs} 224242 \mathrm{I}^{\#}(\mathrm{~A} / \mathrm{G})$ & $42.3 / 42.3 / 15.4$ & $46.2 / 38.5 / 15.4$ & 0.964 & 36.5 & 34.6 & 0.955 \\
\hline rs I697405" (G/A) & $51.9 / 42.3 / 5.8$ & $46.2 / 38.5 / 7.7$ & 0.920 & 26.9 & 26.9 & 1.000 \\
\hline $\mathrm{rs} 1697406^{\#}(\mathrm{~A} / \mathrm{G})$ & $67.3 / 30.8 / 1.9$ & 69.2/23.I/0 (F:7.7) & 0.858 & 17.3 & 11.5 & 1.000 \\
\hline \multicolumn{7}{|l|}{$N R 4 A 2$} \\
\hline rsl $2803(G / A)$ & $69.2 / 28.8 / 1.9$ & $61.5 / 38.5 / 0$ & 0.721 & 16.3 & 19.2 & 0.704 \\
\hline \multicolumn{7}{|l|}{ PTHLH } \\
\hline rs2796" (A/G) & $73.1 / 25.0 / 1.9$ & $84.6 / 15.4 / 0$ & 0.589 & 14.4 & 7.7 & 0.672 \\
\hline $\mathrm{rs} 6253^{\#}(\mathrm{~A} / \mathrm{G})$ & $32.7 / 51.9 / 15.4$ & $23.1 / 76.9 / 0$ & 0.296 & 41.3 & 38.5 & 0.656 \\
\hline rs6245" (A/G) & $80.8 / 19.2 / 0$ & $92.3 / 7.7 / 0$ & 0.439 & 9.6 & 3.8 & 1.000 \\
\hline
\end{tabular}

Notes: Significant result is marked with bold type. F means failure rate (\%) to genotype analysis. "SNPs are located in $3^{\prime}$ untranslated region and analyzed in 65 patients (responder: 52, nonresponder: 13).

Abbreviations: $\mathrm{AA}$, homozygote major allele; $\mathrm{AB}$, heterozygote allele; $\mathrm{BB}$, homozygote minor allele; SNP, single nucleotide polymorphism.

frequently found in the Asian population (MAF: Han Chinese $52.3 \%$, Japanese $55.8 \%)^{54}$ results in different functionality ${ }^{55}$ and showed the consistent result with the previous study by Rothe et al. ${ }^{26}$ The study suggested that an Asian dialysis patient carrying a homozygous $(\mathrm{G} / \mathrm{G})$ variant of $C A S R$ rs1042636 showed significant iPTH reduction after 2-month cinacalcet treatment compared to another six patients having different genotypes. Several other studies ${ }^{27-29}$ on $C A S R$ also presented the possible association of CASR rs 1042636 with PTH level difference. However, the functionality of rs 1802757 which is located in $3^{\prime}$ untranslated region (UTR) has not been well documented. We can assume that the rs1802757 influences the microRNAs which have the binding sites within 3'UTR, and can decrease gene expression of various mRNAs. ${ }^{56,57}$ Other studies presented that CaSR activated by calcimimetics decreased PTH-mRNA stability through the posttranslational modification of the PTH-mRNA binding protein AUF $1 .^{58}$ The rs1802757 can be investigated further via similar mechanism. In particular, the SNPs of rs1042636 and rs1802757 and haplotypes of GCC and ATT occurred at high frequencies of $45.8 \%, 35.9 \%, 52.9 \%$, and $37.5 \%$, respectively; in our result, the impact of these variants should not be negligible among Asians.

The regulation of PTH secretion and synthesis involves a multitude of pathways. ${ }^{59}$ However, in our study, the SNPs of genes other than CASR seemed not to correlate with cinacalcet response. Studies of Casr and $V d r$ knockout mice can explain part of our results. VDR-deficient mice which developed severe SHPT could be corrected by stabilizing serum calcium concentrations, ${ }^{60}$ whereas CaSR-deficient mice with SHPT were not corrected by elevated serum calcium and vitamin D levels and normal serum phosphorus, ${ }^{61}$ 
Table 5 Association of PTH regulation by cinacalcet with SNPs of CASR, VDR, FGFRI, KL, ALPL, RGSI4, NR4A2, and PTHLH

\begin{tabular}{|c|c|c|c|c|c|}
\hline \multirow[t]{3}{*}{ Gene/SNP } & \multicolumn{3}{|l|}{ Genotype model* } & \multirow{3}{*}{$\frac{\frac{\text { Dominant model } * *}{\text { BB }+ \text { AB vs AA }}}{\text { OR }(95 \% \mathrm{Cl})}$} & \multirow{3}{*}{$\begin{array}{l}\text { Recessive model }{ }^{* *} \\
\text { BB vs } A B+A A \\
\text { OR }(95 \% \mathrm{CI})\end{array}$} \\
\hline & BB & AB & $\mathbf{A A}$ & & \\
\hline & OR (95\% Cl) & OR $(95 \% \mathrm{Cl})$ & OR & & \\
\hline \multicolumn{6}{|l|}{$\overline{C A S R}$} \\
\hline \multirow[t]{2}{*}{ rs $1042636(A / G)$} & $0.074(0.008-0.721)$ & $0.395(0.106-1.479)$ & I & $0.267(0.074-0.957)$ & $0.139(0.017-1.142)$ \\
\hline & $P=0.025$ & $P=0.168$ & & $P=0.035$ & $P=0.050$ \\
\hline \multirow[t]{2}{*}{ rs222I266 (C/T) } & $5.500(0.839-36.059)$ & $3.826(0.730-20.044)$ & I & $4.613(0.945-22.526)$ & $2.813(0.758-10.431)$ \\
\hline & $P=0.076$ & $P=0.112$ & & $P=0.065$ & $P=0.142$ \\
\hline \multirow[t]{2}{*}{ rs10190(C/T) } & $5.500(0.74 I-40.803)$ & $4.304(0.730-20.044)$ & I & $4.033(0.8 \mathrm{II}-20.056)$ & $1.929(0.423-8.784)$ \\
\hline & $P=0.095$ & $P=0.112$ & & $P=0.109$ & $P=0.405$ \\
\hline \multirow[t]{2}{*}{ rs $1802757(C / T)$} & $8.625(1.077-69.075)$ & $3.680(0.707-19.156)$ & I & $4.362(0.878-21.669)$ & $3.600(0.695-18.646)$ \\
\hline & $P=0.042$ & $P=0.122$ & & $P=0.056$ & $P=0.136$ \\
\hline \multicolumn{6}{|l|}{$V D R$} \\
\hline \multirow[t]{2}{*}{ rs7975232 (G/T) } & & $0.365(0.073-1.823)$ & I & $0.324(0.065-1.607)$ & \\
\hline & & $P=0.219$ & & $P=0.200$ & \\
\hline \multirow[t]{2}{*}{ rs2228570 (C/T) } & $0.500(0.050-4.978)$ & $1.440(0.416-4.983)$ & I & $1.212(0.362-4.059)$ & $0.402(0.046-3.497)$ \\
\hline & $P=0.554$ & $P=0.565$ & & $P=0.755$ & $P=0.672$ \\
\hline \multirow[t]{2}{*}{ rsI5444I0 (A/G) } & & $0.402(0.046-3.497)$ & I & $0.402(0.046-3.497)$ & \\
\hline & & $P=0.409$ & & $P=0.672$ & \\
\hline \multirow[t]{2}{*}{ rsll574I29(T/C) } & & $0.438(0.050-3.853)$ & I & $0.350(0.04 I-3.015)$ & \\
\hline & & $P=0.456$ & & $P=0.439$ & \\
\hline \multirow[t]{2}{*}{ rs3847987 (G/T) } & & $0.74 I(0.14 I-3.905)$ & I & $0.606(0.118-3.121)$ & \\
\hline & & $P=0.723$ & & $P=0.717$ & \\
\hline \multirow[t]{2}{*}{ rs739837 (C/A) } & & $0.917(0.245-3.433)$ & I & $0.815(0.220-3.021)$ & \\
\hline & & $P=0.897$ & & $P=1.000$ & \\
\hline \multirow[t]{2}{*}{ rs9729 (C/A) } & & $1.007(0.268-3.791)$ & I & $0.889(0.239-3.307)$ & \\
\hline & & $P=0.991$ & & $P=1.000$ & \\
\hline FGFRI & & & & & \\
\hline rs|3317 (C/T) & $1.500(0.355-6.347)$ & $0.429(0.100-1.828)$ & I & $0.724(0.229-2.286)$ & $2.045(0.520-8.044)$ \\
\hline & $P=0.582$ & $P=0.252$ & & $P=0.58 \mathrm{I}$ & $P=0.442$ \\
\hline$K L$ & & & & & \\
\hline rsI $207568(C / T)$ & & $1.029(0.304-3.486)$ & I & $0.972(0.289-3.276)$ & \\
\hline & & $P=0.963$ & & $P=0.964$ & \\
\hline RGSI4 & & & & & \\
\hline rs4074995 (A/G) & $0.500(0.053-4.732)$ & $0.938(0.267-3.292)$ & I & $0.818(0.252-2.653)$ & $0.512(0.057-4.626)$ \\
\hline & $P=0.546$ & $P=0.920$ & & $P=0.738$ & $P=1.000$ \\
\hline ALPL & & & & & \\
\hline rsI69742I (A/G) & $1.82 I(0.266-12.473)$ & $2.705(0.497-14.7 \mid 8)$ & I & $2.36 \mid(0.465-11.979)$ & $0.929(0.220-3.928)$ \\
\hline & $P=0.54$ & $P=0.250$ & & $P=0.484$ & $P=1$ \\
\hline rs3200254 (C/T) & $0.667(0.104-4.261)$ & I. $120(0.282-4.449)$ & I & $0.973(0.26 I-3.627)$ & $0.62 I(0.121-3.197)$ \\
\hline & $P=0.668$ & $P=0.872$ & & $P=1.000$ & $P=0.718$ \\
\hline rs2242420 (C/T) & & $0.972(0.260-3.632)$ & I & $0.915(0.246-3.400)$ & \\
\hline & & $P=0.967$ & & $P=1.000$ & \\
\hline rs224242I (A/G) & $0.917(0.153-5.508)$ & $0.833(0.22 \mathrm{I}-3.138)$ & I & $0.856(0.252-2.902)$ & $1.000(0.186-5.390)$ \\
\hline & $P=0.924$ & $P=0.788$ & & $P=0.802$ & $P=1.000$ \\
\hline rsI697405 (G/A) & $1.500(0.132-17.037)$ & I.227 (0.347-4.343) & I & $1.080(0.308-3.791)$ & I.485 (0.14I-15.659) \\
\hline & $P=0.744$ & $P=0.75 \mid$ & & $P=0.904$ & $P=0.574$ \\
\hline rsl697406 (A/G) & & $1.200(0.3 \mid 3-4.596)$ & I & $0.686(0.164-2.866)$ & \\
\hline & & $P=0.790$ & & $P=0.739$ & \\
\hline$N R 4 A 2$ & & & & & \\
\hline rsI2803 (G/A) & & I.500 (0.422-5.338) & I & $\mathrm{I} .250(0.36 \mathrm{I}-4.327)$ & \\
\hline & & $P=0.531$ & & $P=0.724$ & \\
\hline PTHLH & & & & & \\
\hline rs2796 (A/G) & & $0.487(0.096-2.472)$ & I & $0.452(0.090-2.280)$ & \\
\hline & & $P=0.385$ & & $P=0.488$ & \\
\hline rs6253 (A/G) & & I.286 (0.34I-4.853) & I & $1.000(0.268-3.732)$ & \\
\hline & & $P=0.7 I I$ & & $P=1.000$ & \\
\hline & & $0.350(0.04 I-3.0 I 5)$ & & $0.350(0.04 I-3.0 I 5)$ & \\
\hline rs6245 (A/G) & & $P=0.339$ & 1 & $P=0.439$ & \\
\hline
\end{tabular}

Notes: Significant results are marked in bold type. *Logistic regression analysis. **Chi-square analysis.

Abbreviations: $\mathrm{AA}$, homozygote major allele; $\mathrm{AB}$, heterozygote; $\mathrm{BB}$, homozygote minor allele; $\mathrm{Cl}$, confidence interval; OR, odds ratio; PTH, parathyroid hormone; SNP, single nucleotide polymorphism. 
A

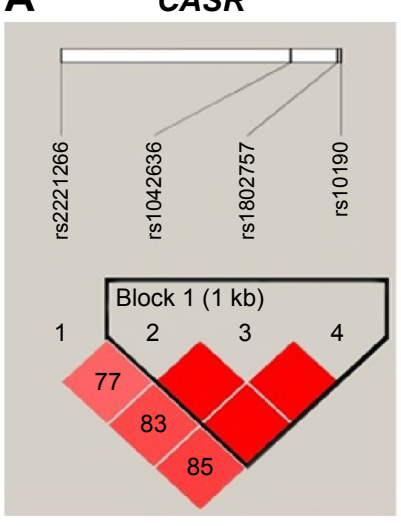

B

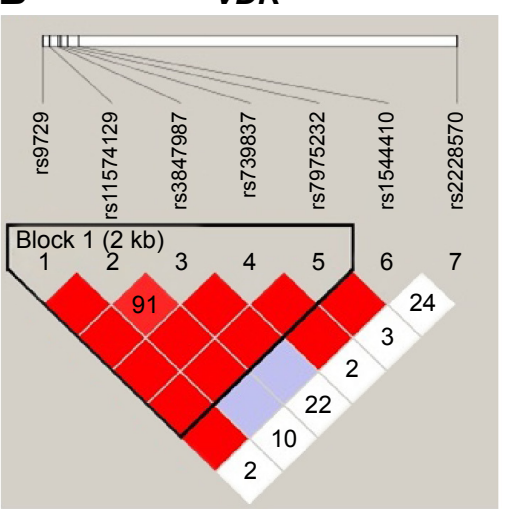

C

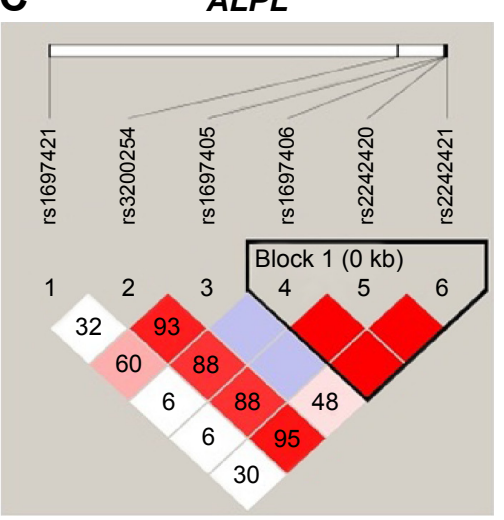

Figure I The haplotype blocks of the three studied genes: (A) CASR, (B) VDR, and (C) ALPL.

Notes: The numbers indicate the extent of linkage equilibrium based on $D^{\prime}$ value between two SNPs calculated with Haploview 4.2. Only CASR haplotype of GCC (odds ratio [OR]: 0.355 [0.15I-0.832], $P=0.015$ ) and ATT (OR: 2.769 [I.208-6.347], $P=0.014$ ) showed the significance $D^{\prime}$ represents the linkage disequilibrium of one haplotype.

Abbreviation: SNPs, single nucleotide polymorphisms.

which demonstrates that $\mathrm{CaSR}$ is the major regulator of $\mathrm{PTH}$ secretion and bone abnormalities. ${ }^{62}$ Thus, other genes related to phosphate and bone metabolism do not have much effect on the PTH regulation by cinacalcet.

The maximum dose of cinacalcet approved in Korea is $100 \mathrm{mg} /$ day and the higher titration dose did not control the PTH level in nonresponders. Previous studies suggested that early response to cinacalcet determined the effectiveness of cinacalcet treatment. ${ }^{63,64}$

Therefore, our study can suggest that CKD patients who carry $\mathrm{T}$ allele of rs 1802757 and/or ATT haplotype of rs1042646, rs10190 and rs1802757 have high risk of cinacalcet treatment failure and might be candidates for the parathyroidectomy. There have been pharmacoeconomic issues about the indefinite long-term cinacalcet treatment versus one-time surgery. The research in the 5 European countries by Iannazzo et $\mathrm{a}^{65}$ proved that cinacalcet treatment was beneficial when compared to the standard therapy of vitamin D analogues in cost-effectiveness and cost utility model compared to standard therapy of vitamin D analogs. Komaba et $\mathrm{l}^{66}$ presented that cinacalcet was cost-effective in $99.9 \%$ of the Monte Carlo simulations in parathyroidectomy ineligible patients, but only $0.9 \%$ of the simulations in parathyroidectomy eligible patients. Thus, nonresponders to cinacalcet treatment can have minimal cost-effectiveness compared to surgery in economic issues.

SHPT patients are highly vulnerable to cardiovascular morbidity and mortality and neither parathyroidectomy ${ }^{67}$ nor cinacalcet ${ }^{68}$ reduces the risk of death or major cardiovascular events in SHPT patients undergoing dialysis. Therefore, the

Table 6 Association of PTH regulation by cinacalcet with CASR, VDR, and ALPL haplotypes

\begin{tabular}{|c|c|c|c|c|c|}
\hline Gene/SNP & Haplotype & $\begin{array}{l}\text { Haplotype } \\
\text { frequency (\%) }\end{array}$ & $\begin{array}{l}\text { Case/control } \\
\text { frequencies (\%) }\end{array}$ & OR $(95 \% \mathrm{Cl})$ & $P$-value \\
\hline \multicolumn{6}{|l|}{ CASR } \\
\hline rsI042636 (A/G) & GCC & 0.529 & $33.3 / 58.5$ & $0.355(0.15 \mathrm{I}-0.832)$ & 0.015 \\
\hline rs I $802757(\mathrm{C} / \mathrm{T})$ & ATT & 0.375 & $57.3 / 31.9$ & $2.769(1.208-6.347)$ & 0.014 \\
\hline \multirow[t]{2}{*}{ rsI0I90(C/T) } & ACC & 0.064 & $8.5 / 5.8$ & $1.786(0.420-7.597)$ & 0.423 \\
\hline & ACT & 0.032 & $0.9 / 3.8$ & - & 0.575 \\
\hline \multicolumn{6}{|l|}{ VDR } \\
\hline rs9729 (C/A) & CTGCG & 0.815 & $88.5 / 79.8$ & $1.940(0.53 \mid-7.08 I)$ & 0.404 \\
\hline rsII574I 29 (T/C) & АCTAT & 0.092 & $7.7 / 9.6$ & $0.783(0.16 \mid-3.814)$ & I \\
\hline rs3847987 (G/T) & ATGAT & 0.068 & $3.8 / 7.6$ & $0.480(0.057-4.019)$ & 0.686 \\
\hline \multicolumn{6}{|l|}{ rs739837 (C/A) } \\
\hline \multicolumn{6}{|l|}{ rs7975232 (G/T) } \\
\hline \multicolumn{6}{|l|}{$A L P L$} \\
\hline rsl697406 (A/G) & GCG & 0.638 & $65.4 / 63.5$ & $1.088(0.442-2.678)$ & 0.855 \\
\hline rs2242420 (C/T) & GCA & 0.192 & $23.1 / 18.3$ & I.342 (0.475-3.794) & 0.578 \\
\hline rs224242I (A/G) & ATA & 0.162 & $11.5 / 17.3$ & $0.623(0.169-2.300)$ & 0.567 \\
\hline
\end{tabular}

Note: Significant results are marked in bold type.

Abbreviations: $\mathrm{Cl}$, confidence interval; OR, odds ratio; PTH, parathyroid hormone; SNP, single nucleotide polymorphism. 
timely management of SHPT at the initial stage may offer long-term survival in CKD patients. Several studies have proved the efficacy and safety of cinacalcet in stage 3 and 4 CKD patients with SHPT; ${ }^{69-72}$ however, the noticeable PTH decrease followed by hypocalcaemia and hyperphosphatemia brought about the avoidance of cinacalcet prescription in CKD predialysis patients. ${ }^{73-75}$ Since our findings in this study provided a potential target for the investigation of cinacalcet nonresponder group who have high possibility of parathyroidectomy or refractory SHPT, CASR SNPs may be useful biomarkers to administer cinacalcet in early stage of CKD patients to attain the individualized medical treatment of SHPT.

The main factors of cinacalcet resistance after several months or years of therapy are severely enlarged parathyroid gland $(>1 \mathrm{~cm})$, nodular hyperplasia, ${ }^{22,76,77}$ and the downregulation of CaSR and VDR by uremic status which is resulted by inadequate dialysis. ${ }^{78}$ However, the parathyroid hyperplasia did not affect the cinacalcet nonresponsiveness in our shortterm study as in previous studies. ${ }^{79,80}$ The dose of vitamin D was not different between two groups and findings from the study by Block et $\mathrm{al}^{81}$ presented that cinacalcet reduced PTH levels regardless of whether the vitamin D doses were increased, decreased, or unchanged. We performed multiple testing by Bonferroni correction and false discovery rate test. The result turned out that the association of rs1042636 or rs 1802757 with PTH change was not significant. However, neither of these methods take into account the correlation of SNPs due to linkage disequilibrium, which tags the genetic variation across gene regions. Our result showed that the three SNPs of CASR, five SNPs of VDR, and three SNPs of $A L P L$ comprise one haploblock each, representing that the SNPs are not independent and highly correlated. Thus, we rather not reflect the multiple testing in the results.

Our study has several limitations as follows. The first, 70 Korean patients are relatively small for genetic association study and diverse ethnic factors could not be considered. This limitation should warrant further large-scale studies to replicate and confirm our findings. The second, the included patients were on either hemodialysis or peritoneal dialysis; the $\mathrm{Kt} / \mathrm{V}\left(\mathrm{K}_{\text {urea }} \times \mathrm{T}_{\mathrm{d}} / \mathrm{V}_{\text {urea }}\right)$ value which indicates the dialysis adequacy is not recirpocally conversed between two modalities, so the influence of dialysis could not be evaluated in our study. The third, we focused on the 3-month treatment of cinacalcet, and the result of long-term follow-up study on the mineral bone disease and cardiovascular disease could not be assessed. The last, most patients were on either phosphate binders or vitamin $\mathrm{D}$ analogs and the proportion of patients on either drug was not different between two groups, but the possible impact of different amount of concomitant calcium intake cannot be ruled out.

\section{Conclusion}

We obtained supporting evidence for an association between cinacalcet response and CASR polymorphisms. We performed a comprehensive search for SNPs within genes associated with $\mathrm{PTH}$ regulations to find the precise frequencies and association with cinacalcet response in 70 dialytic SHPT patients for the first time in Korea. CASR SNPs rs1802757, rs1042636, and haplotypes of rs1042636, rs10190, and rs1802757 were significantly associated with cinacalcet response variance.

\section{Acknowledgments}

This research was supported by the Basic Science Research Program through the National Research Foundation of Korea (NRF) funded by the Ministry of Education (2014R1A1A2055734) and BK21 Plus Program in 2015.

\section{Disclosure}

The authors report no conflicts of interest in this work.

\section{References}

1. Delmez JA, Slatopolsky E. Hyperphosphatemia: its consequences and treatment in patients with chronic renal disease. Am J Kidney Dis. 1992;19:303-317.

2. Winchester JF, Rotellar C, Goggins M, Robino D, Rakowski TA, Argy WP. Calcium and phosphate balance in dialysis patients. Kidney Int Suppl. 1993;41:S174-S178.

3. Levin A, Thompson CR, Ethier J, et al. Left ventricular mass index increase in early renal disease: impact of decline in hemoglobin. Am J Kidney Dis. 1999;34:125-134.

4. Harnett JD, Kent GM, Barre PE, Taylor R, Parfrey PS. Risk factors for the development of left ventricular hypertrophy in a prospectively followed cohort of dialysis patients. J Am Soc Nephrol. 1994: 1486-1490.

5. Locatelli F, Cannata-Andia J, Drueke T, et al. Management of disturbances of calcium and phosphate metabolism and chronic renal insufficiency with emphasis on the control of hyperphosphatemia. Nephrol Dial Transplant. 2002;17:723-731.

6. Padhi D, Harris R. Clinical pharmacokinetic and pharmacodynamic profile of cinacalcet hydrochloride. Clin Pharmacokinet. 2009;48(5):303-311.

7. Drueke TB. Modulation and action of the calcium sensing receptor. Nephrol Dial Transplant. 2004;19(suppl 5):V20-V26.

8. Nagano N, Nemeth EF. Functional proteins involved in regulation of intracellular $\mathrm{Ca}(21)$ for drug development: the extracellular calcium receptor and an innovative medical approach to control secondary hyperparathyroidism by calcimimetics. J Pharmacol Sci. 2005;97: 355-360.

9. National Kidney Foundation:K/DOQI Clinical Practice Guidelines for Bone Metabolism and Disease in Chronic Kidney Disease. Am J Kidney Dis. 2003;42(suppl 3):S1-S201.

10. Quarles LD. Cinacalcet HCl: a novel treatment for secondary hyperparathyroidism in stage 5 chronic kidney disease. Kidney Int Suppl. 2005;68(Supplement 96):s24-s28. 
11. Cunningham J, Danese M, Olson K, Klassen P, Chertow G. Effects of the calcimimetic cinacalcet $\mathrm{HCl}$ on cardiovascular disease, fracture, and health-related quality of life in secondary hyperparathyroidism. Kidney Int. 2005;68(4):1793.

12. Kruse A, Eisenberger U, Frey F, Mohaupt M. The calcimimetic cinacalcet normalizes serum calcium in renal transplant patients with persistent hyperparathyroidism. Nephrol Dial Transplant. 2005;20:1311-1314.

13. Yang R, Freeman K, Reinke C, et al. Tertiary hyperparathyroidism in kidney transplant recipients: characteristics of patients selected for different treatment strategies. Transplantation. 2012;94:70-76.

14. Goodman W, Hladik G, Turner S, et al. The calcimimetic agent AMG 073 lowers plasma parathyroid hormone levels in hemodialysis patients with secondary hyperparathyroidism. J Am Soc Nephrol. 2002;13: $1017-1024$.

15. Wüthrich R, Martin D, Bilezikian J. The role of calcimimetics in the treatment of hyperparathyroidism. Eur J Clin Invest. 2007;37:915-922.

16. Ballinger AE, Palmer SC, Nistor I, Craig JC, Strippoli GF. Calcimimetics for secondary hyperparathyroidism in chronic kidney disease patients. Cochrane Database of Syst Rev. 2014(12):1-82.

17. Kuczera $P$, Adamczak M, Więcek A. Safety and efficiency of treatment with cinacalcet of haemodialysed patients with chronic kidney disease and secondary hyperparathyroidism. Endokrynol Pol. 2013;64(3): $176-181$.

18. Li D, Shao L, Zhou H, Jiang W, Zhang W, Xu Y. The efficacy of cinacalcet combined with conventional therapy on bone and mineral metabolism in dialysis patients with secondary hyperparathyroidism: a meta-analysis. Endocrine. 2013;43(1):68-77.

19. Quarles LD, Sherrard DJ, Adler S, et al. The calcimimetic AMG 073 as a potential treatment for secondary hyperparathyroidism of end-stage renal disease. J Am Soc Nephrol. 2003;14:575-583.

20. Lafrance J, Cardinal H, Leblanc M, et al. Effect of cinacalcet availability and formulary listing on parathyroidectomy rate trends. BMC Nephrol. 2013;14:100.

21. Wetmore J, Gurevich K, Sprague S, et al. A randomized trial of cinacalcet versus vitamin D analogs as monotherapy in secondary hyperparathyroidism (PARADIGM). Clin J Am Soc Nephrol. 2015;10(6): 1031-1040.

22. Okada M, Tominaga Y, Izumi K, et al. Tertiary hyperparathyroidism resistant to cinacalcet treatment. Ther Apher Dial. 2011;15(Suppl 1): 33-37.

23. Gogusev J, Duchambon P, Hory B, et al. Depressed expression of calcium receptor in parathyroid gland tissue of patients with hyperparathyroidism. Kidney Int. 1997;51(1):328-336.

24. Fukuda N, Tanaka H, Tominaga Y, Fukagawa M, Kurokawa K, Seino Y. Decreased 1,25-dihydroxyvitamin D3 receptor density is associated with a more severe form of parathyroid hyperplasia in chronic uremic patients. J Clin Invest. 1993;92:1436-1442.

25. Kifor O, Moore FJ, Wang P, et al. Reduced immunostaining for the extracellular Ca2+-sensing receptor in primary and uremic secondary hyperparathyroidism. J Clin Endocrinol Metab. 1996;81:1598-1606.

26. Rothe HM, Shapiro WB, Sun WY, Chou SY. Calcium-sensing receptor gene polymorphism Arg990Gly and its possible effect on response to cinacalcet HCl. Pharmacogenet Genomics. 2005;15:29-34.

27. Yano S, Sugimoto T, Kanzawa M, et al. Association of polymorphic alleles of the calcium-sensing receptor gene with parathyroid hormone secretion in hemodialysis patients. Nephron. 2000;85(4):317-323.

28. Yokoyama K, Shigematsu T, Tsukada T, et al. Calcium-sensing receptor gene polymorphism affects the parathyroid response to moderate hypercalcemic suppression in patients with end-stage renal disease. Clin Nephrol. 2002;57(2):131-135.

29. Eren P, Turan K, Berber I, et al. The clinical significance of parathyroid tissue calcium sensing receptor gene polymorphisms and expression levels in end-stage renal disease patients. Clin Nephrol. 2009;72(2): 114-121.

30. Chudek J, Karkoszka H, Schmidt-Gayk H, Ritz E, Kokot F. Plasma parathyroid hormone, phosphatemia and vitamin $\mathrm{D}$ receptor genotype: are they interrelated? J Nephrol. 2000;13(1):54-58.
31. Yokoyama K, Shigematsu T, Tsukada T, et al. Apa I polymorphism in the vitamin $\mathrm{D}$ receptor gene may affect the parathyroid response in Japanese with end-stage renal disease. Kidney Int. 1998;53(2):454-458.

32. Ozdemir FN, Sezer S, Atac B, et al. Vitamin D receptor BsmI and TagI gene polymorphisms in a Turkish ESRD population: influences on parathyroid hormone response. Transplant Proc. 2005;37(7): 2922-2924.

33. Management of secondary hyperparathyroidism and mineral metabolism abnormalities in dialysis patients. Wolters Kluwer; 2015. Available from: www.uptodate.com/contents/managementof-secondary-hyperparathyroidism-and-mineral-metabolism-abnormalities-in-dialysis-patients?source=search_result\&search=treatme $\mathrm{nt}+$ failure+secondary+hyperparathyroidism\&selectedTitle $=1 \sim 150$. Accessed December 9.

34. Cunningham J, Locatelli F, Rodriguez M. Secondary hyperparathyroidism: pathogenesis, disease progression, and therapeutic options. Clin J Am Soc Nephrol. 2011;6:913-921.

35. Yamauchi M, Sugimoto T, Yamaguchi T, et al. Association of polymorphic alleles of the calcium-sensing receptor gene with the clinical severity of primary hyperparathyroidism. Clin Endocrinol (Oxf). 2001; 55:373-379

36. Filopanti M, Verga U, Ermetici F, et al. MEN1-related hyperparathyroidism: response to cinacalcet and its relationship with the calciumsensing receptor gene variant Arg990Gly. Eur J Endocrinol. 2012; 167(2):157-164.

37. Han G, Wang O, Nie M, et al. Clinical phenotypes of Chinese primary hyperparathyroidism patients are associated with the calciumsensing receptor gene R990G polymorphism. Eur J Endocrinol. 2013; 169(5):629-638.

38. Yokoyama K, Shigematsu T, Kagami S, et al. Vitamin D receptor gene polymorphism detected by digestion with Apa I influences the parathyroid response to extracellular calcium in Japanese chronic dialysis patients. Nephron. 2001;89(3):315-320.

39. Karkoszka H, Chudek J, Strzelczyk P, et al. Vitamin D receptor gene polymorphism and the rate of bone loss of the femur neck and lumbar spine in hemodialized patients with chronic renal failure. Pol Merkur Lekarski. 1998;28:199-202.

40. Jun JK, Kim SM. Association study of fibroblast growth factor 2 and fibroblast growth factor receptors gene polymorphism in Korean ossification of the posterior longitudinal ligament patients. J Korean Neurosurg Soc. 2012;52:7-13.

41. Guimarães JM, Guimarães IC, Duarte ME, et al. Polymorphisms in BMP4 and FGFR1 genes are associated with fracture non-union. J Ortho Res. 2013;31(12):1971-1979.

42. Sakan H, Nakatani K, Asai O, et al. Reduced renal $\alpha$-Klotho expression in CKD patients and its effect on renal phosphate handling and vitamin D metabolism. PLoS One. 2014;9(1):e86301.

43. Kestenbaum B, Glazer NL, Köttgen A, et al. Common genetic variants associate with serum phosphorus concentration. J Am Soc Nephrol. 2010; 21(7):1223-1232.

44. Yasui T, Okada A, Urabe Y, et al. A replication study for three nephrolithiasis loci at 5q35.3, 7p14.3 and 13q14.1 in the Japanese population. J Hum Genet. 2013;58:588-593.

45. Boron WF, Boulpaep EL. Medical Physiology: A Cellular and Molecular Approach. Philadelphia: Saunders; 2012.

46. Tsui HW, Inman RD, Reveille JD, Tsu FWL. Association of a TNAP haplotype with ankylosing spondylitis. Arthritis Rheum. 2007; 56(1):234-243

47. Cheng N, Cai Q, Fang M, et al. No significant association between genetic polymorphisms in the TNAP gene and ankylosing spondylitis in the Chinese Han population. Rheumatol Int. 2008;29(3):305-310.

48. Meir T, Durlacher K, Pan Z, et al. Parathyroid hormone activates the orphan nuclear receptor Nurr1 to induce FGF23 transcription. Kidney Int. 2014;86:1106-1115.

49. Tetradis S, Bezouglaia O, Tsingotjidou A. Parathyroid hormone induces expression of the nuclear orphan receptor Nurr1 in bone cells. Endocrinology. 2001;142(2):663-670. 
50. Nakajima K, Tamai M, Okaniwa S, et al. Humoral hypercalcemia associated with gastric carcinoma secreting parathyroid hormone: a case report and review of the literature. Endocr J. 2013;60(5):557-562.

51. Tenne M, McGuigan F, Jansson L, et al. Genetic variation in the PTH pathway and bone phenotypes in elderly women: evaluation of $\mathrm{PTH}$, PTHLH, PTHR1 and PTHR2 genes. Bone. 2008;42(4):719-727.

52. Truong NU, deB Edwardes MD, Papavasiliou V, Goltzman D, Kremer R. Parathyroid hormone-related peptide and survival of patients with cancer and hypercalcemia. Am J Med. 2003;115(2):115-121.

53. Iwase M, Takemi T, Manabe M, Nagumo M. Hypercalcemia upon recurrence of renal cell carcinoma producing parathyroid hormonerelated protein. Int J Oral Maxillofac Surg. 2003;32(2):174-180.

54. dbSNP. National Library of Medicine. Available from: http://www. ncbi.nlm.nih.gov/snp/. Accessed December 9, 2015.

55. Miyata T, Miyazawa S, Yasunaga T. Two types of amino acid substitutions in protein evolution. J Mol Evol. 1979;12(3):219-236.

56. Barrett LW, Fletcher S, Wilton SD. Regulation of eukaryotic gene expression by the untranslated gene regions and other non-coding elements. Cell Mol Life Sci. 2012;69(21):3613-3634.

57. Pichon X, Wilson LA, Stoneley M, et al. RNA binding protein/RNA element interactions and the control of translation. Curr Protein Pept Sci. 2012;13(4):294-304.

58. Levi R, Ben Dov I, Lavi-Moshayoff V, et al. Increased parathyroid hormone gene expression in secondary hyperparathyroidism of experimental uremia is reversed by calcimimetics: correlation with posttranslational modification of the trans acting factor AUF1. J Am Soc Nephrol. 2006;17:107-112.

59. Gilbert SJ, Weiner DE, Gipson DS, Perazella MA, Tonelli M. National Kidney Foundation's Primer on Kidney Disease. 6th ed. Philadelphia: Elsevier; 2014.

60. Li Y, Amling M, Pirro A, et al. Normalization of mineral ion homeostasis by dietary means prevents hyperparathyroidism, rickets and osteomalacia, but not alopecia in vitamin $\mathrm{D}$ receptor ablated mice. Endocrinology. 1998;139:4391-4396.

61. Garner S, Pi M, Tu Q, Quarles L. Rickets in cation sensing receptor deficient mice: an unexpected skeletal phenotype. Endocrinology. 2001; 142(9):3996-4005.

62. Block GA. The impact of calcimimetics on mineral metabolism and secondary hyperparathyroidism in end-stage renal disease. Kidney Int Suppl. 2003;64(Suppl 87):S131-S136.

63. Torres PS, Utiel FJB, Perales MCS, Cortés MJG, Baldán MMB, Bañasco VP. Analysis of efficacy and factors that impact the response of secondary hyperparathyroidism to cinacalcet in haemodialysis patients. Nefrologia. 2010;30(4):443-451.

64. Kim JK, Kwon YJ, Choi KH, et al. Rapid decrease of intact parathyroid hormone could be a predictor of better response to cinacalcet in hemodialysis patients. Yonsei Med J. 2013;54(2):453-463.

65. Iannazzo S, Carsi M, Chiroli S. A cost-utility analysis of cinacalcet in secondary hyperparathyroidism in five European countries. Appl Health Econ Health Policy. 2012;10(2):127-138.

66. Komaba H, Moriwaki K, Goto S, et al. Cost-effectiveness of cinacalcet hydrochloride for hemodialysis patients with severe secondary hyperparathyroidism in Japan. Am J Kidney Dis. 2012;60(2):262-271.
67. Conzo G, Perna AF, Savica V, et al. Impact of parathyroidectomy on cardiovascular outcomes and survival in chronic hemodialysis patients with secondary hyperparathyroidism. A retrospective study of 50 cases prior to the calcimimetics era. BMC Surg. 2013;13(Suppl 2):S2-S4.

68. EVOLVE Investigators, Chertow GM, Block GA, et al. Effect of cinacalcet on cardiovascular disease in patients undergoing dialysis. N Engl J Med. 2012;367(26):2482-2494.

69. Charytan C, Coburn JW, Chonchol M, et al. Cinacalcet hydrochloride is an effective treatment for secondary hyperparathyroidism in patients with CKD not receiving dialysis. Am J Kidney Dis. 2005; 46:58-67.

70. Chonchol M, Locatelli F, Abboud HE, et al. A randomized, doubleblind, placebo-controlled study to assess the efficacy and safety of cinacalcet $\mathrm{HCl}$ in participants with CKD not receiving dialysis Am J Kidney Dis. 2009;53:197-207.

71. Montenegro J, Cornago I, Gallardo I, et al. Efficacy and safety of cinacalcet for the treatment of secondary hyperparathyroidism in patients with advanced chronic kidney disease before initiation of regular dialysis. Nephrology. 2012;17:26-31.

72. de Francisco AL, Piñera C, Palomar R. Cinacalcet should be used to treat secondary hyperparathyroidism in stage 3-4 chronic kidney disease. Nat Clin Pract Nephrol. 2008;4:366-367.

73. Coyne DW. Cinacalcet should not be used to treat secondary hyperparathyroidism in stage 3-4 chronic kidney disease. Nat Clin Pract Nephrol. 2008;4:364-365.

74. Cannata-Andía JB, Fernández-Martín JL. Mineral metabolism: should cinacalcet be used in patients who are not on dialysis? Nat Rev Nephrol. 2009;5:307-308.

75. Kalantar-Zadeh K, Kovesdy CP. Is it worth correcting hyperparathyroidism if hyperphosphatemia and hypocalcemia worsen? A cinacalcet story. Am J Kidney Dis. 2009;53:183-188.

76. Shoback D, Bilezikian J, Binder T, Graves T, Turner S, Peacock M. Calcimimetic AMG 073 normalizes total serum calcium in patients with primary hyperparathyroidism [abstract]. J Bone Miner Res. 2000; $15: 210$.

77. Indridason OS, Heath H, Khosla S, Yohay DA, Quarles LD. Nonsuppressible parathyroid hormone secretion is related to gland size in uremic secondary hyperparathyroidism. Kidney Int. 1996;50:1663-1671.

78. Lewin E, Garfia B, Recio F, Rodriguez M, Olgaard K. Persistent downregulation of calcium-sensing receptor $\mathrm{mRNA}$ in rat parathyroids when severe secondary hyperparathyroidism is reversed by an isogenic kidney transplantation. J Am Soc Nephrol. 2002;13(8):2110-2116.

79. Komaba H, Nakanishi S, Fujimori A, et al. Cinacalcet effectively reduces parathyroid hormone secretion and gland volume regardless of pretreatment gland size in patients with secondary hyperparathyroidism. Clin J Am Soc Nephrol. 2010;5:2305-2314.

80. Meola M, Petrucci I, Barsotti G. Long-term treatment with cinacalcet and conventional therapy reduces parathyroid hyperplasia in severe secondary hyperparathyroidism. Nephrol Dial Transplant. 2009; 24:982-989.

81. Block G, Martin K, de Francisco A, et al. Cinacalcet for secondary hyperparathyroidism in patients receiving hemodialysis. $N$ Engl J Med. 2004;350(15):1516-1525. 


\section{Supplementary materials}

Table SI The primer sets and melting temperature (Tm) used for the SNaPshot assay

\begin{tabular}{|c|c|c|c|c|c|}
\hline \multirow{2}{*}{$\begin{array}{l}\text { Gene } \\
\text { CASR }\end{array}$} & \multirow{2}{*}{$\begin{array}{l}\text { rs number } \\
\text { rs } 1042636\end{array}$} & \multirow{2}{*}{$\begin{array}{l}\text { Strand } \\
\text { Forward }\end{array}$} & \multicolumn{2}{|c|}{ Primer sequence } & \multirow{2}{*}{$\begin{array}{l}\mathrm{Tm} \\
55\end{array}$} \\
\hline & & & Forward primer & CAGATGCAAGCAGAAGGT & \\
\hline & & & Reverse primer & CAAAGCTCTGTGAACTGGA & \\
\hline & & & Genotyping primer & GCCTCAGAAGAAYKCCATGGCSCAC & \\
\hline & rs2221266 & Reverse & Forward primer & CTGTTTACCCCTGTTGGAT & 55 \\
\hline & & & Reverse primer & TCCATTGTGTGCTACGTTT & \\
\hline & & & Genotyping primer & GTGTGCTACGTTTGAGGTAAT & \\
\hline & rs 10190 & Reverse & Forward primer & CCAAGAAAGATCCACCCTCA & 55 \\
\hline & & & Reverse primer & TGGGCTAGGCAGTACAATCTG & \\
\hline & & & Genotyping primer & atccacataagagctctgaggggaa & \\
\hline & rs|802757 & Forward & Forward primer & CCAAGAAAGATCCACCCTCA & 55 \\
\hline & & & Reverse primer & TGGGCTAGGCAGTACAATCTG & \\
\hline & & & Genotyping primer & ggagccagMgagacaRaccggggtt & \\
\hline \multirow[t]{21}{*}{ VDR } & rs7975232 & Forward & Forward primer & aagtcactggagggcttt & 55 \\
\hline & & & Reverse primer & TTAGCTTCATGCTGCACTC & \\
\hline & & & Genotyping primer & TGGTGGGATTGAGCAGTGAGG & \\
\hline & rs2228570 & Reverse & Forward primer & gatgcaacatctgaaacca & 55 \\
\hline & & & Reverse primer & AGCCTTCACAGGTCATAGC & \\
\hline & & & Genotyping primer & agggaagtgctggecRccattKcctcc & \\
\hline & rsI5444I0 & Forward & Forward primer & CTACTTTGCTGGTTTGCAG & 55 \\
\hline & & & Reverse primer & ATTGCCTCCAAAATCAATC & \\
\hline & & & Genotyping primer & TTCCTGGRGCCACAGACAGRCCTGC & \\
\hline & rsII574I29 & Forward & Forward primer & TGTTCACCCACAGGGCTAA & 55 \\
\hline & & & Reverse primer & CACAGCTTCCAGGAGTTGG & \\
\hline & & & Genotyping primer & GAGTGATACAACTTCAAGTGCATGC & \\
\hline & rs3847987 & Reverse & Forward primer & CACCCAGCCCATTCTCTCT & 55 \\
\hline & & & Reverse primer & GGGAAGAAAACCCACCTCA & \\
\hline & & & Genotyping primer & AAGGGGGTGGGGTGGGAGCTGTGGG & \\
\hline & rs739837 & Reverse & Forward primer & CACCCAGCCCATTCTCTCT & 55 \\
\hline & & & Reverse primer & GGGAAGAAAACCCACCTCA & \\
\hline & & & Genotyping primer & CTCAACATCAGTCAGCAGCCACTTA & \\
\hline & rs9729 & Forward & Forward primer & agcaggtaccactgatttgga & 60 \\
\hline & & & Reverse primer & gtctcgaactcccgacctc & \\
\hline & & & Genotyping primer & TGCTAAACGAGTcaatcceMtcatt & \\
\hline \multirow[t]{3}{*}{ FGFRI } & rs 13317 & Forward & Forward primer & aattatccaggcatggtg & 55 \\
\hline & & & Reverse primer & CCCAACAAATACAGTCTGGT & \\
\hline & & & Genotyping primer & TCTATGTGGGCATGGTTTTGCC & \\
\hline \multirow[t]{3}{*}{$K L$} & rs|207568 & Reverse & Forward primer & CTCACCTAAGGGGGAATC & 55 \\
\hline & & & Reverse primer & ACGCTCAGGTTCATTCTCT & \\
\hline & & & Genotyping primer & cggggagaaaaggcgcSgaccaacttt & \\
\hline \multirow[t]{3}{*}{ RGSI4 } & rs4074995 & Forward & Forward primer & GGAATGGAGAGAAGTGGAC & 55 \\
\hline & & & Reverse primer & GAGGGTGGAAGAGCTGTAG & \\
\hline & & & Genotyping primer & CATTTCATGCCAGCAGCTACGTTTCTC & \\
\hline \multirow[t]{3}{*}{ SLC34AI* } & rs38I2035 & Forward & Forward primer & CATCCCCTTTCTGGGACAG & 55 \\
\hline & & & Reverse primer & ccaggTTGCAAGTCCTCAA & \\
\hline & & & Genotyping primer & TCССTTCСAACСTGTTCCСATTGAC & \\
\hline \multirow[t]{9}{*}{ ALPL } & rsl69742I & Forward & Forward primer & TTGGAGTGCAGTGGTGTGA & 55 \\
\hline & & & Reverse primer & GCAGCTCCCAAAGAAGAGG & \\
\hline & & & Genotyping primer & CССTTATGAGCACTTCTTAAAGGCA & \\
\hline & rs3200254 & Forward & Forward primer & ACCAGGCTGGGAAAGTGTC & 55 \\
\hline & & & Reverse primer & TCTGGTATCCCCTCCAAGG & \\
\hline & & & Genotyping primer & CACCTGGAAGAGCTTCAAACCGAGA & \\
\hline & rs2242420 & Reverse & Forward primer & ACTACGTCCCCCACGTGAT & 60 \\
\hline & & & Reverse primer & CCTGGTGGTCTTGGAGTGA & \\
\hline & & & Genotyping primer & AGAGGTAAGGAGATGGGAGTGGGA & \\
\hline
\end{tabular}


Table SI (Continued)

\begin{tabular}{|c|c|c|c|c|c|}
\hline \multirow[t]{2}{*}{ Gene } & \multirow{2}{*}{$\begin{array}{l}\text { rs number } \\
\text { rs224242I }\end{array}$} & \multirow{2}{*}{$\begin{array}{l}\text { Strand } \\
\text { Forward }\end{array}$} & \multicolumn{2}{|c|}{ Primer sequence } & \multirow{2}{*}{$\begin{array}{l}\mathrm{Tm} \\
60\end{array}$} \\
\hline & & & Forward primer & ACTACGTCCCCCACGTGAT & \\
\hline & & & Reverse primer & CCTGGTGGTCTTGGAGTGA & \\
\hline & & & Genotyping primer & ССТCTGGAACCCCCCAGGCCCTACA & \\
\hline & rsI697405 & Reverse & Forward primer & ACTACGTCCCCCACGTGAT & 60 \\
\hline & & & Reverse primer & CCTGGTGGTCTTGGAGTGA & \\
\hline & & & Genotyping primer & GGCCCCCACСTCССTGCCССTTGAK & \\
\hline & rsI697406 & Forward & Forward primer & ACTACGTCCCCCACGTGAT & 60 \\
\hline & & & Reverse primer & CCTGGTGGTCTTGGAGTGA & \\
\hline & & & Genotyping primer & CTGCAACTGCAAGAAAGGGGACCCA & \\
\hline \multirow[t]{3}{*}{ NR4A2 } & rs $\mid 2803$ & Reverse & Forward primer & GGCAGAGATAGCCGTGTGA & 55 \\
\hline & & & Reverse primer & TGGGCTAGGCAGTACAATCTG & \\
\hline & & & Genotyping primer & atccacataagagctctgagggggaa & \\
\hline \multirow[t]{9}{*}{ PTHLH } & rs2796 & Forward & Forward primer & TGCTCTCAGCTGGGTTTTG & 55 \\
\hline & & & Reverse primer & CATGATGCTGTTCTGCAGTGT & \\
\hline & & & Genotyping primer & TCAGAATATTGTCTGCCTTAAAGCA & \\
\hline & rs6253 & Forward & Forward primer & TGCTCTCAGCTGGGTTTTG & 55 \\
\hline & & & Reverse primer & CATGATGCTGTTCTGCAGTGT & \\
\hline & & & Genotyping primer & TCTTCATAATTTGCTGGAGAAGTGT & \\
\hline & rs6245 & Forward & Forward primer & TGCTCTCAGCTGGGTTTTG & 55 \\
\hline & & & Reverse primer & CATGATGCTGTTCTGCAGTGT & \\
\hline & & & Genotyping primer & TCCAGCACCATAGAGAGGCGYTAGA & \\
\hline
\end{tabular}

Note: *SLC34A4 was not included in the final analysis because it didn't meet the Hardy Weinberg Equilibrium (HWE). 


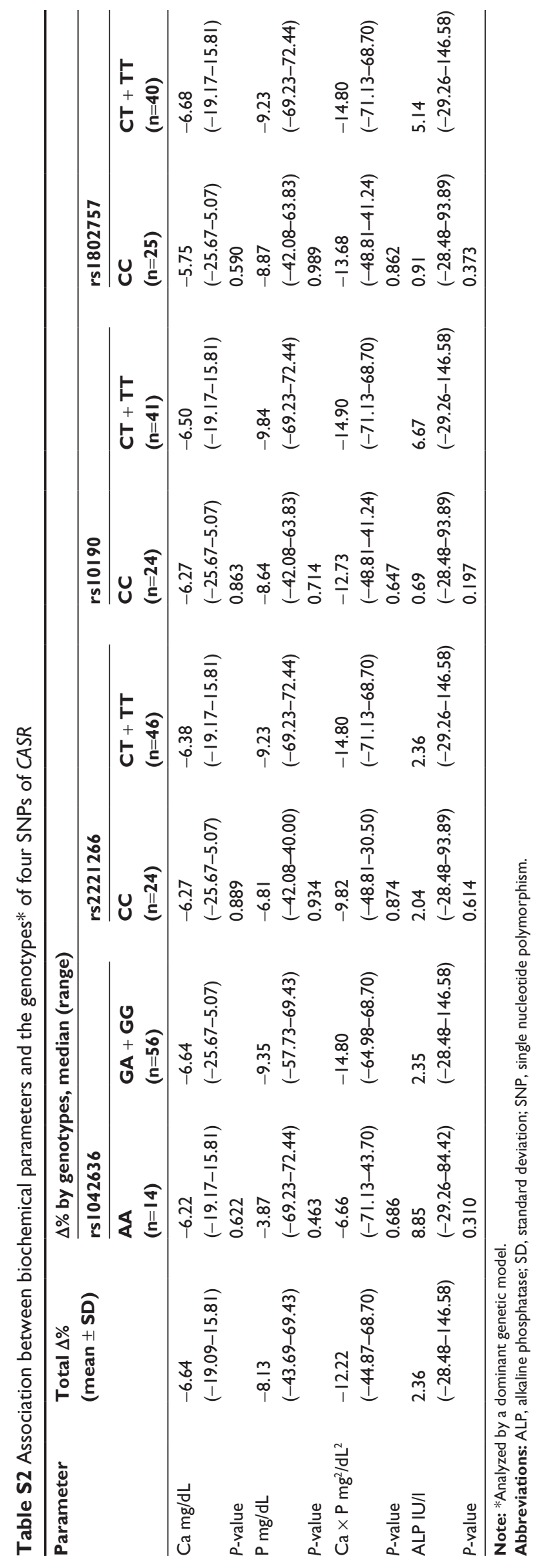




\section{Publish your work in this journal}

Drug Design, Development and Therapy is an international, peerreviewed open-access journal that spans the spectrum of drug design and development through to clinical applications. Clinical outcomes, patient safety, and programs for the development and effective, safe, and sustained use of medicines are a feature of the journal, which has also been accepted for indexing on PubMed Central. The manuscript management system is completely online and includes a very quick and fair peer-review system, which is all easy to use. Visit http://www.dovepress.com/testimonials.php to read real quotes from published authors.

Submit your manuscript here: http://www.dovepress.com/drug-design-development-and-therapy-journal 